\title{
THE GROOVED LIP EFFECT ON RECIPROCATING HYDRAULIC ROD SEAL PERFORMANCES IN TRANSIENT CONDITION: ELASTOHYDRODYNAMIC LUBRICATION
}

\author{
Y. BAHI* \\ Moulay Ismail UniversityHigh School of engineering ENSAM Meknes - Morocco \\ Research Laboratory in Advanced Materials and Applications - LEM2A \\ Research team: High-tech materials and energetic systems - MASEN \\ E-mail: y.bahi@ensam-umi.ac.ma \\ M. El GADARI \\ Moulay Ismail UniversityHigh School of engineering ENSAM Meknes - Morocco \\ Research Laboratory in Mechanical, Mechatronic \& Control - L2MC \\ Research team: multidisciplinary engineering and mechatronics system - IMSM \\ E-mail:m.elgadari@ensam-umi.ac.ma \\ M. RAHMOUNE \\ Moulay Ismail University High School of Technology EST Meknes - Morocco \\ Research Laboratory in Advanced Materials and Applications - LEM2A \\ Research team: High-tech materials and energetic systems - MASEN \\ E-mail: rahmoune@umi.ac.ma
}

\begin{abstract}
It is commonly known that the sealing performance of dynamic seals is significantly influenced by the surface finish. To reduce friction effect and leakage ratio, new generations of grooved lip or shaft have emerged, but only two computational models were performed up to now with a textured elastomeric lip: spiral groove in the axial direction or micro-cavities according to the circumferential direction. However, if the numerical results have confirmed the slight effect of the grooved lip on the rotary lip seal performances, it seems relevant to investigate the influence of such grooves on the reciprocating hydraulic rod seal behavior.

Thus, the scope of this work is to perform a parametric study of the grooved lip throughout a one-dimensional elastohydrodynamic model by taking into account the elasticity of the lip and the shaft roughness.

After confirming the validity of the current model, numerical simulations have been performed and compared with experiments. The effect of lip grooves on the hydraulic rod seal behavior in outstroke and instroke shaft motion has been underlined. Thereby, it is shown that the leakage and the average film thickness are sensible to both the depth and the density of the lip groove. Additionally, a slight effect of the pattern shape is observed on the friction force.
\end{abstract}

Key words: hydraulic rod seal, surface-textured, roughness, friction force, leakage.

\section{Introduction}

The $\mathrm{U}$ cup hydraulic rod seal is the machine component the most used to prevent leakage with a minimal wear effect for a hydraulic cylinder. From the sixties, large importance has been devoted to modeling hydraulic seals. Because of the behavior complexity of such a device, several physical phenomena can interfere. This includes the thermal effect of viscous friction, which impacts simultaneously the elasticity of the elastomeric seal and the rheological law of the lubricant film.

\footnotetext{
* To whom correspondence should be addressed
} 
However, to predict the film thickness behavior, several approaches have been performed. We noticed in this paper only two numerical methods:

- inverse Hydrodynamic Lubrication (IHL): By assuming the hydrodynamic pressure is equal to static pressure simulated on commercial packaging software (FEM structural analysis), the Reynolds equation is solved with film thickness as an unknown parameter as carefully detailed by Crudu thesis [3]. The numerical results agreed slightly with measurements;

- elastohydrodynamic lubrication (EHL): Elgadari et al. [4] have performed recently this approach by resolving the Reynolds equation and taking into account the seal elasticity, the roughness of the lip and the shaft.

It is important to note that previously, Lawrie et al. [1] have demonstrated by experimentations the presence of a thin film throughout lubricated contact. They underlined that in the outstroke motion the film is thicker and the lip shape has a substantial effect on sealing performances of the hydraulic U-cup seal. Besides, by studying the roughness rod effect on rectangular polyurethane seal performances, Vissher et al. [2] have demonstrated that beyond a critical value of the rod arithmetic roughness the seal could leak.

An earlier numerical study was performed by Kanters [5] to predict the sealing mechanism of the reciprocating rod seal. The model was assumed axisymmetric with stationary and isothermal conditions. Experimentations have agreed to simulations and showed that the roughness impacts significantly the seal performance.

It is generally accepted that the mixed lubrication raises the wear effect and thus reduces the seal lifetime. However, Sandor et al. [6] have proved that the full film lubricated zone could also increase the friction force of an operational hydraulic seal. Recently, a numerical model of reciprocating seals has been developed by Salant et al. [7]. The results for rod seals show that they operate with mixed lubrication and that the seal roughness is the most important parameter to prevent leakage. This work confirmed the transient numerical model performed by Azam et al. [8] where the rod velocity variation was considered. This study showed that films are thinner during the outstroke comparing to the instroke case.

Nowadays, to enhance the seal lifetime, a new generation of the textured shaft has emerged. This improvement was demonstrated numerically by Elgadari et al. [4, 9]. Indeed, the leakage rate and friction were reduced by using an appropriate rod groove. Thereby, a judicious texture could improve the wear behavior of such a device. Also, Xiaohong et al. [10], have presented a numerical study to determine the optimal geometry of lip seal patterns to reduce leakage and friction. Therefore, Zhang et al. [11] have performed a numerical method to analyze the textured rod effects on seal wear. The proposed numerical analysis method was confirmed by comparing the simulation results with those obtained experimentally. Based on the simulation results, the authors demonstrated that the seal lifetime is substantially depending on the depth pattern in the axial direction.

Further, Kligerman et al. [12] have proposed an elastohydrodynamic model of the elastomeric hydraulic seal to investigate the textured surface effect. The authors have pointed out that patterns with tapered edges increase significantly the hydrodynamic lift. A similar numerical analysis was performed by Huang et al [13] for a reciprocating hydraulic cylinder seal with a textured rod. The results indicate that the friction force cannot be significantly reduced by texturing the rod, and may even be slightly increased.

If an appropriate grooved shaft decreases the friction force as described previously in $[4,9]$, it is relevant to check how far the elastomeric lip grooves impact the sealing performances.

Thus, the present work investigates the effect of those grooves on friction force and leakage through a one-dimensional model with full film lubrication in transient conditions [4]. Thereafter, to perform this study, the following steps are proposed:

- comparing the current numerical results "EHL" and friction force measurements,

- investigating the effect of the amplitude and the density of the seal grooves on friction force,

- analyzing the influence of the groove shape on sealing performances. 


\section{Model and validation}

\subsection{Assumptions}

Figure 1 shows a schematic diagram of a typical reciprocating rod seal, while Fig. 2 shows the region near the sealing zone, assuming that:

- the seal operates at a steady speed with reciprocating motion: instroke and outstroke cases,

- the viscosity of the lubricant is constant since the temperature dependence of viscosity is neglected and the piezo-viscosity property is considered and given by the equation:

$$
\mu=\mu_{0} \cdot \exp \left(\alpha \cdot p_{s}\right)
$$

where:

$$
\alpha=\left[34.95+9.65 \log _{10}\left(\mu_{0}\right)\right] \cdot 10^{-9}
$$

- the lubricant side of the seal is flooded with lubricant with a sealed pressure " $p_{s}$ ",

- the average film thickness is uniform in the axial direction, based on previous numerical and experimental results,

- the asperity contact is not considered in this full film lubrication model,

- circumferential shear deformation is assumed according to the equation.

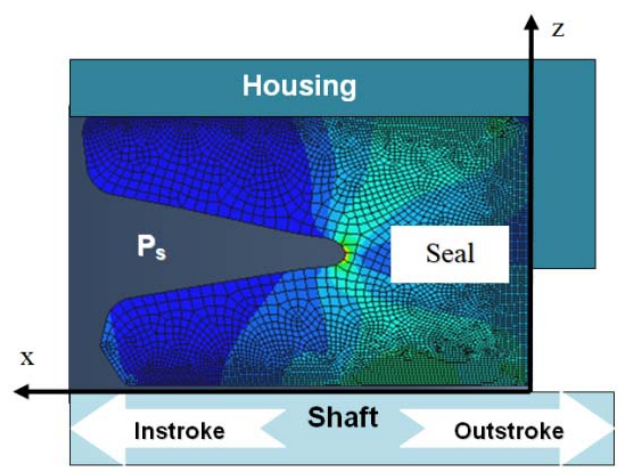

Fig.1. U-Cup reciprocating rod seal.

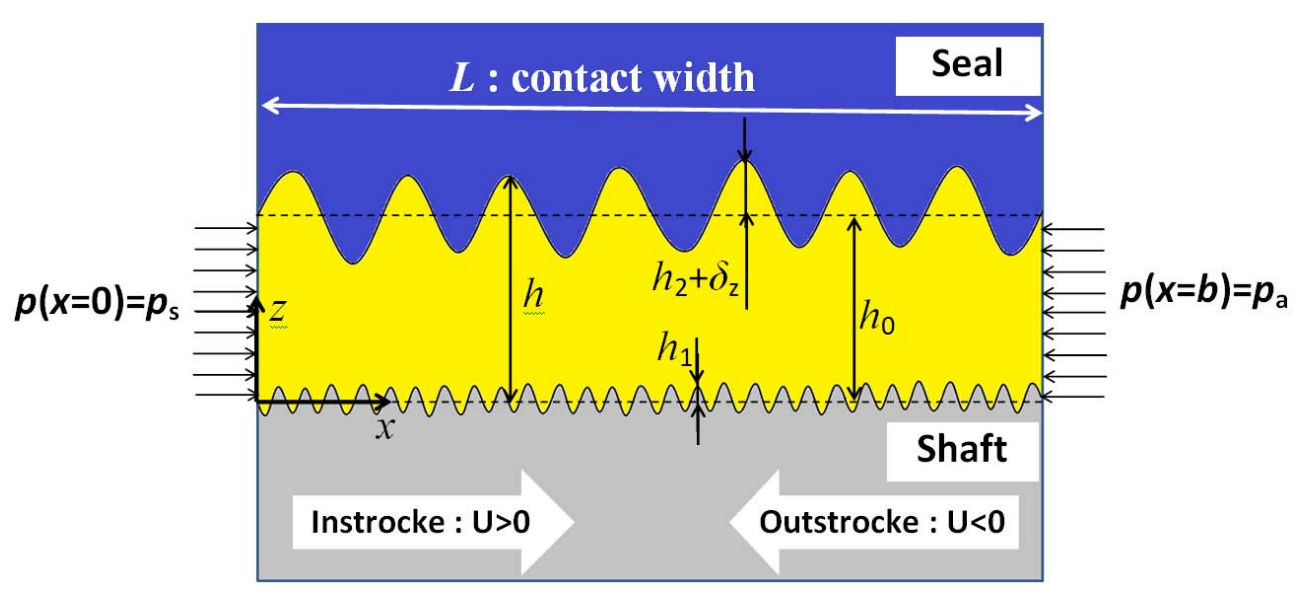

Fig.2. Schematic diagram of the sealing zone. 


\subsection{Governing equations} formulated

To take into the account cavitation effect, modified Reynolds equation is used as Elgadari et al. [4]

$$
F \frac{\partial}{\partial x}\left(h^{3} \frac{\partial D}{\partial x}\right)=6 \mu U \cdot \frac{\partial h}{\partial x}+12 \mu \frac{\partial h}{\partial t}+6 \mu(1-F)\left(U \cdot \frac{\partial D}{\partial x}+2 \frac{\partial D}{\partial t}\right)
$$

When: $D>0, F=1$ and $D=p$, else: $F=0$ and $D=r-h$ where: $r=\frac{\rho}{\rho_{0}} h, \rho$, and $\rho_{0}$ are densities of lubricant-gas mixture and lubricant respectively. $D$ at $x=0$ is equal to pair and at $x=L, D$ is equal to $p_{s}$.

In the structural mechanic analysis, two parameters are to be determinate:

- pressure field " $p c$ " and contact width " $L$ " due to mounting the seal on the shaft (the interference),

- the radial and tangential compliance matrix " $C_{z}$ " and " $C_{x}$ " respectively, according to a study performed by Elgadari et al. [4]. So the elastic formulations are given by

$$
\begin{aligned}
& \left(\delta_{z}\right)_{i}=\sum_{j=1}^{N_{x}}\left(C_{z}\right)_{i, j}\left(p_{j}-p_{c j}\right), \\
& \left(\delta_{x}\right)_{i}=\sum_{j=1}^{N_{x}}\left(C_{x}\right)_{i, j} \tau_{x z j}
\end{aligned}
$$

here $p_{j}$ is the nodal film pressure, $p_{c j}$ is the nodal contact static pressure, $N_{x}$ is the number of nodes, and $\tau_{x z j}$ is the nodal shear stress calculated with

$$
\tau_{x z}=F\left[\frac{1}{2} \frac{\partial p}{\partial x} h+\mu \frac{U}{h}\right]-(1-F) \mu \frac{r U}{h^{2}} .
$$

\subsection{Film thickness}

The symbol $h$ represents film thickness such as

$$
h(x, t)=h_{2}\left(x-\delta_{x}\right)-h_{l}(x-U . t)+h_{0}+\delta_{z}(x, t),
$$

with $h_{1}$ the rod roughness, $h_{2}$ the lip roughness, $h_{0}$ the average film thickness, $\delta_{x}$ and $\delta_{z}$ the axial and normal lip displacement respectively by Eqs (2.4) and (2.5).

To validate the current model, the studies of Crudu [3] and Elgadari [4] are reproduced by simulating 4 sealed pressure cases: $4.5 \mathrm{MPa}, 9.5 \mathrm{MPa}, 12.5 \mathrm{MPa}$, and $19.5 \mathrm{Mpa}$. And considering the similar surface roughness with simple analytical functions.

The lip roughness is assumed sinusoidal and given by

$$
h_{2}\left(x-\delta_{x}\right)=A_{2} \sin \left(\frac{2 \pi}{\lambda_{2}}\left(x-\delta_{x}\right)\right),
$$


and the rod roughness is given by

$$
h_{l}(x-U t)=A_{l} \sin \left(\frac{2 \pi}{\lambda_{l}}(x-U t)\right)
$$

where " $\lambda_{1}$ " and " $\lambda_{2}$ " are the wavelengths according to leakage direction for rod and lip roughness respectively.

\subsection{Validation}

To compare the friction force " $F_{f}$ " to experiments made by Crudu [3], this parameter is calculated by

$$
F_{f}=2 \pi R \int_{0}^{L} \tau_{x z} d x
$$

where $R$ is the shaft radius.

Figure 3 shows that the averaged friction force predicted with the current model gives a good agreement to experiments.

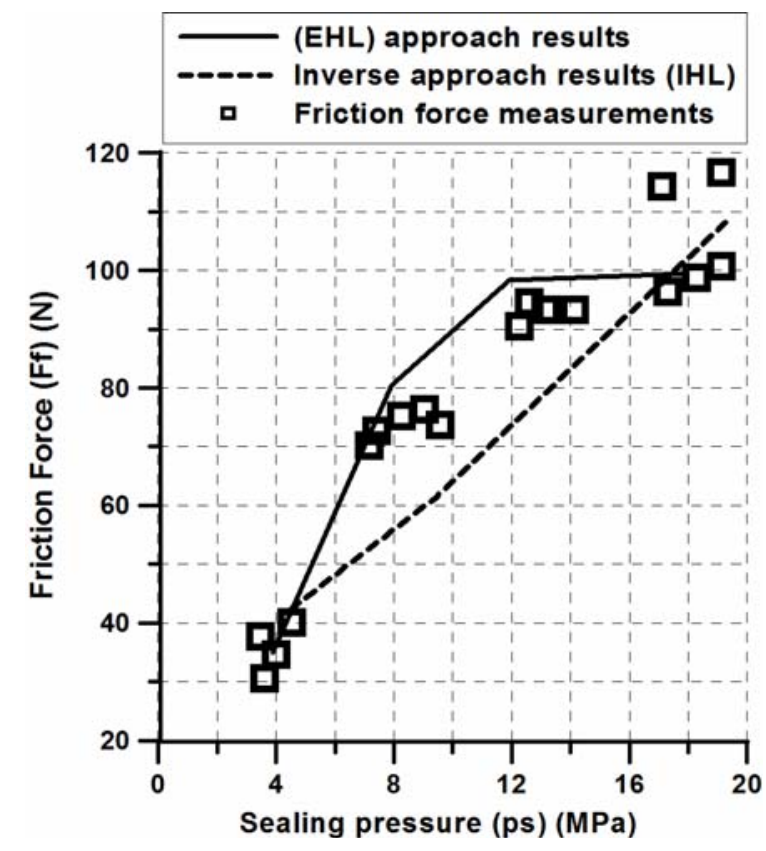

Fig.3. Friction force versus different sealed pressure.

\section{Groove lip effect on sealing performances} Tab.1.

To investigate the effect of the grooved seal, simulations were made with the parameters listed in 
Table1. Parameters adopted for the parametric study.

\begin{tabular}{|c|c|}
\hline Parameter & Numerical value \\
\hline Viscosity & $\mu=1.2610^{-7} \mathrm{MPa} . \mathrm{s}$ \\
\hline Lubricant density (Eq.[4]) & $\rho=974 \mathrm{Kg} / \mathrm{m}^{3}$ \\
\hline Sealed pressure & $p s=5 \mathrm{MPa}$ \\
\hline Rod velocity & $U=+1-80 \mathrm{~mm} / \mathrm{s}$ \\
\hline Groove lip density & $N_{2}$ \\
\hline Lip wavelength roughness & $\lambda_{2}=L / \mathrm{N}_{2}$ \\
\hline Lip roughness fluctuation & $A_{2}$ \\
\hline Rod asperity number & $N_{1}=10$ \\
\hline Rod wavelength roughness & $\lambda_{1}=L / \mathrm{N}_{1}$ \\
\hline Rod roughness fluctuation & $A_{1}=A_{2} / 10$ \\
\hline
\end{tabular}

\subsection{Depth and density effect}

To investigate the effect of depth and density groove on sealing performances, 3 lip surfaces have been considered: by varying the lip amplitude fluctuations $A_{2}$, and the groove periodicity $\lambda_{2}$. Thus, computations have been performed through the 3 following grooved lip (Fig.4) :

- \#J1: Lip groove with $A_{2}=0.752$ microns and $N_{2}=2$ (the grooves are repeated two times $\lambda_{2}=L / 2$ ),

- \#J2: Lip groove with $A_{2}=1.54$ microns and $N_{2}=2$,

- \#J3: Lip groove with $A_{2}=1.54$ microns and $N_{2}=8$ (groove periodicity $\lambda_{2}=L / 8$ ).

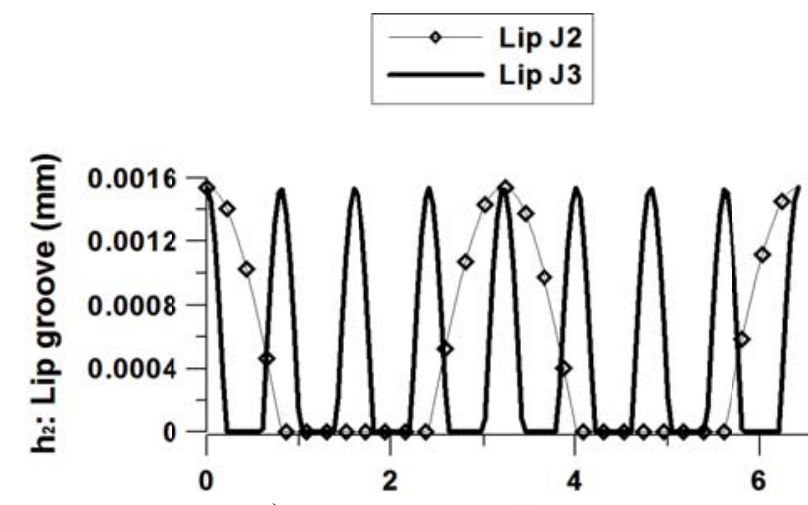

a) $\quad \mathrm{x}$ : Axial direction $(\mathrm{mm})$

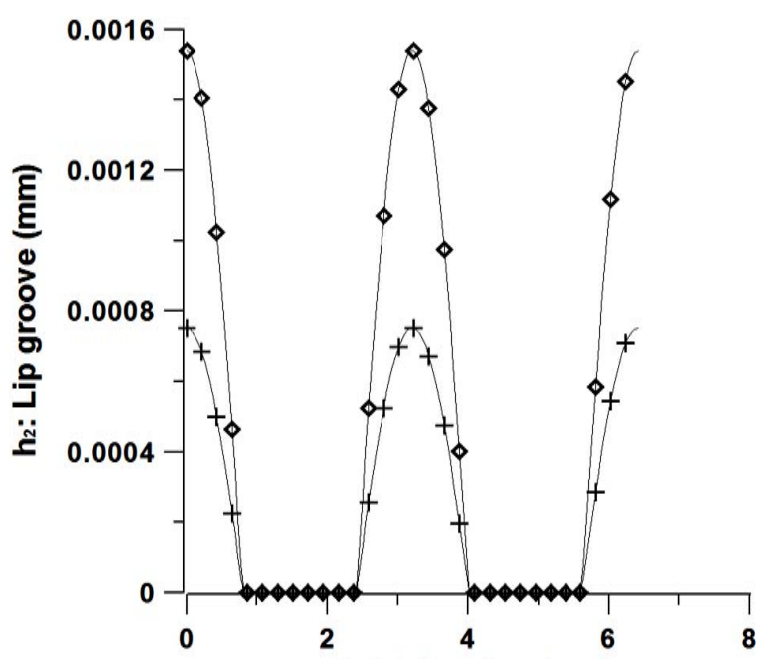

b) $\mathrm{x}$ : Axial direction $(\mathrm{mm})$

Fig.4. Grooved seal: a) By varying the depth, b) By varying the density. 
Figure 5 shows the effect of the amplitude and density on the friction force. Thus, by increasing the groove depth the friction rises substantially and varies slightly by the density.

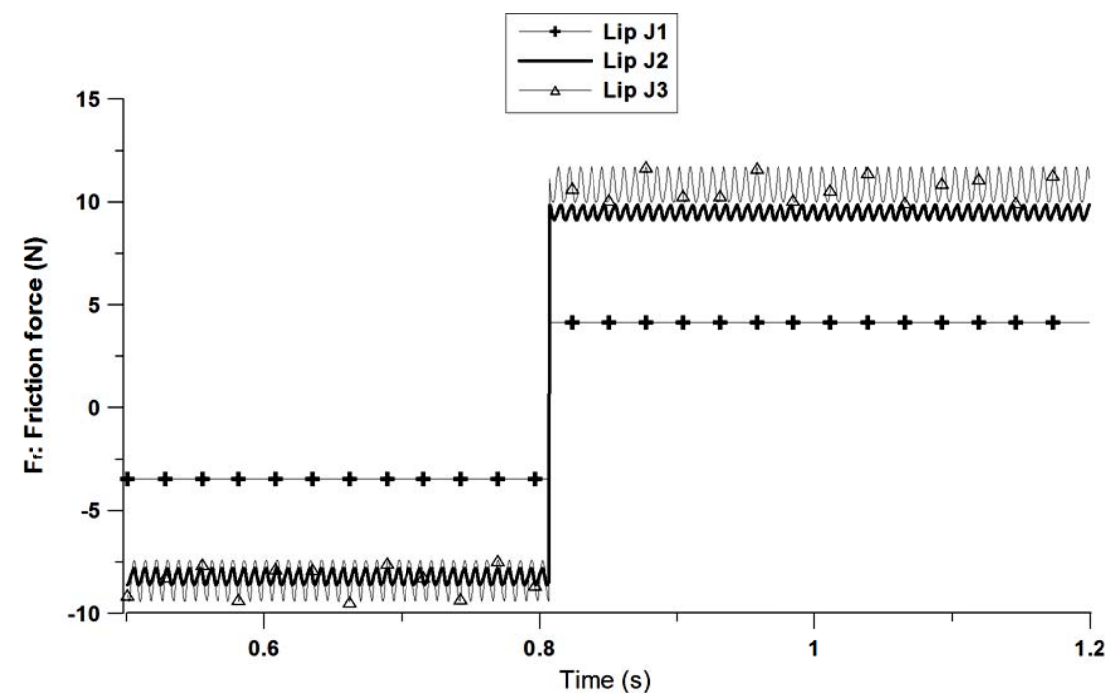

Fig.5. Friction forces in instroke and outstroke cases.

The friction force is more important with deep grooves as confirmed by the tangential lip displacement in Fig.6d. This increase is due to the high pressure produced with the big groove amplitude (Fig.6a) that leads to the rise of the radial lip displacement (Fig.6c) and therefore the film becomes thinner (Fig.6b).

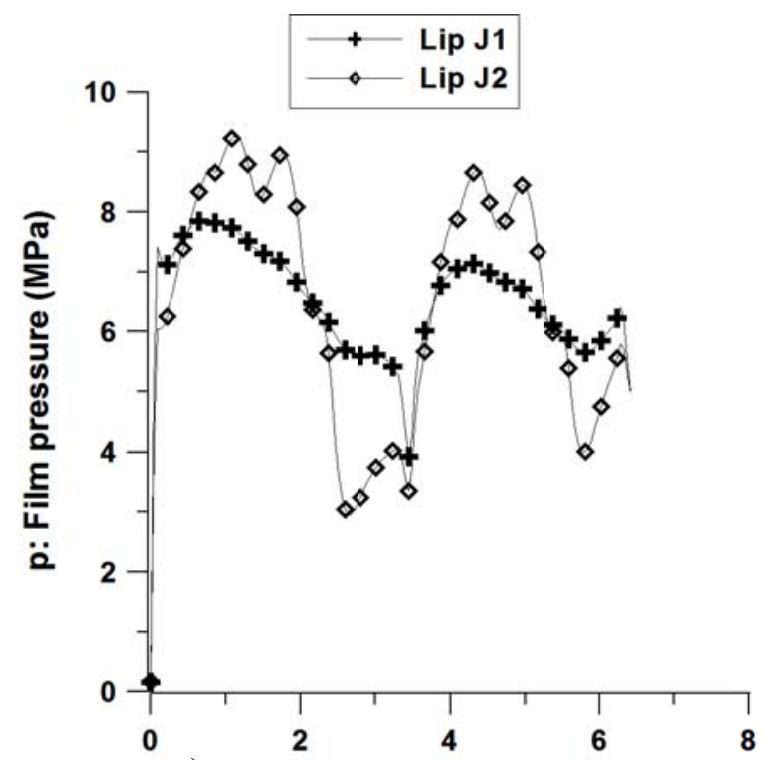

a) $\quad \mathrm{x}$ : Axial direction $(\mathrm{mm})$

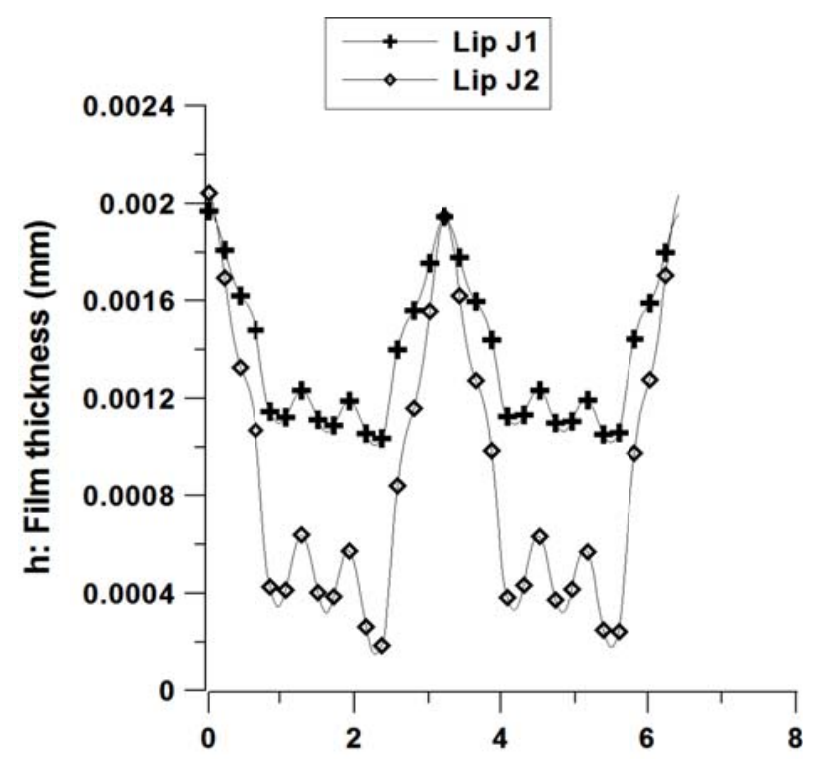

b) $\quad \mathrm{x}$ : Axial direction $(\mathrm{mm})$ 

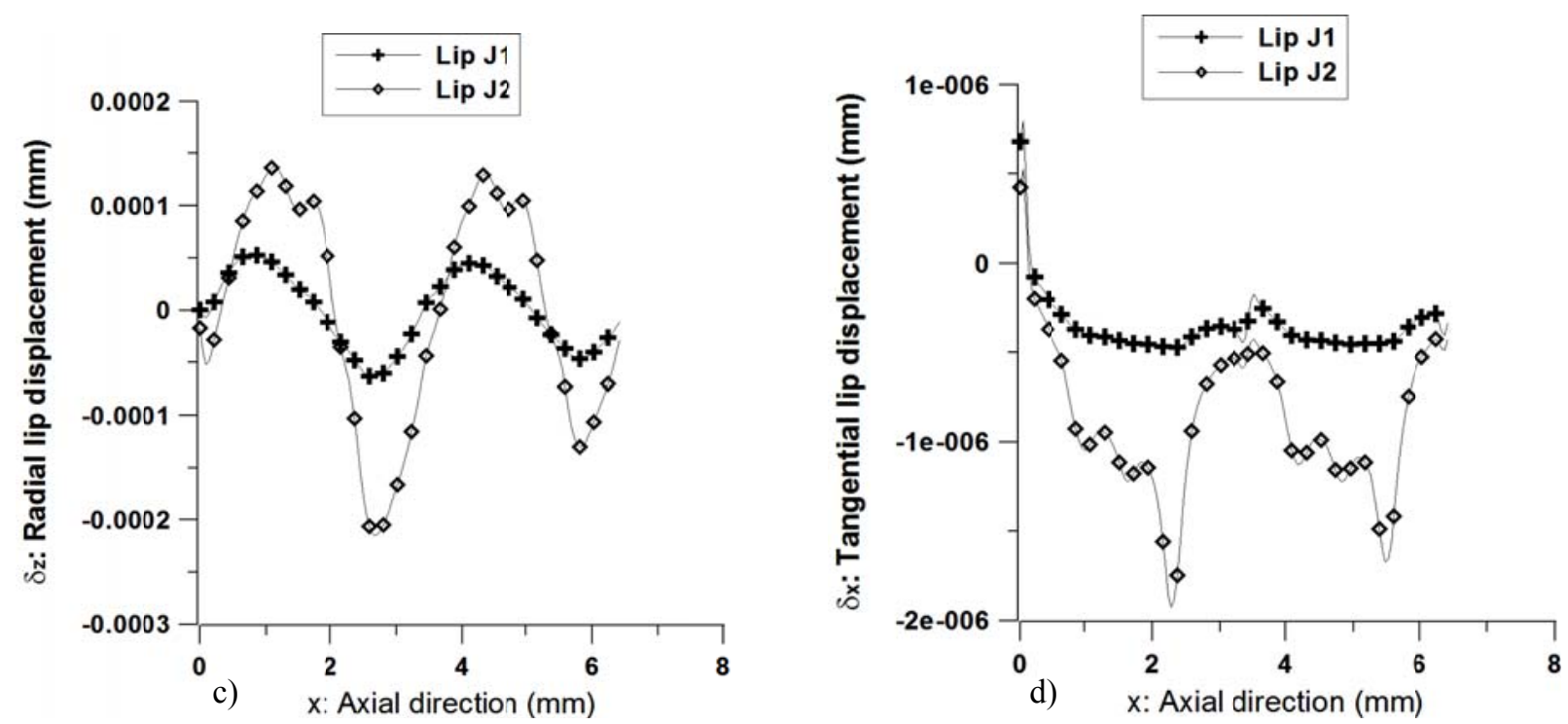

Fig.6. EHL results in instroke case: a) Film pressure, b) Film thickness, c) Radial lip displacement, d) Tangential lip displacement.

\subsection{Shape groove effect}

To study the effect of the groove shape, 2 lip surfaces were studied (Fig.7):

- \#J4: Lip groove with a progressive amplitude given by the equation

$$
h_{2}(x)=A_{2} \frac{x}{L} \sin \left(\frac{2 \pi}{\lambda_{2}} x\right)
$$

- \#J5: Lip groove with a regressive amplitude given by the equation

$$
h_{2}(x)=A_{2} \frac{L-x}{L} \sin \left(\frac{2 \pi}{\lambda_{2}} x\right)
$$

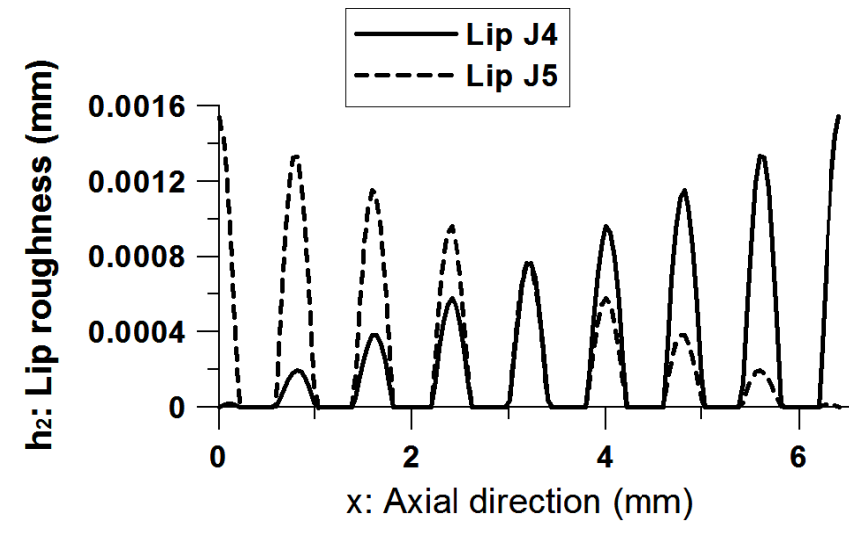

Fig.7. The shape of the grooved seal. 
Figure 8 illustrates the effect of the groove shape of the seal on the friction force. Indeed, by using an appropriate lip pattern (progressive toward the sealed zone) the friction is lower comparing to the regressive or constant amplitude.

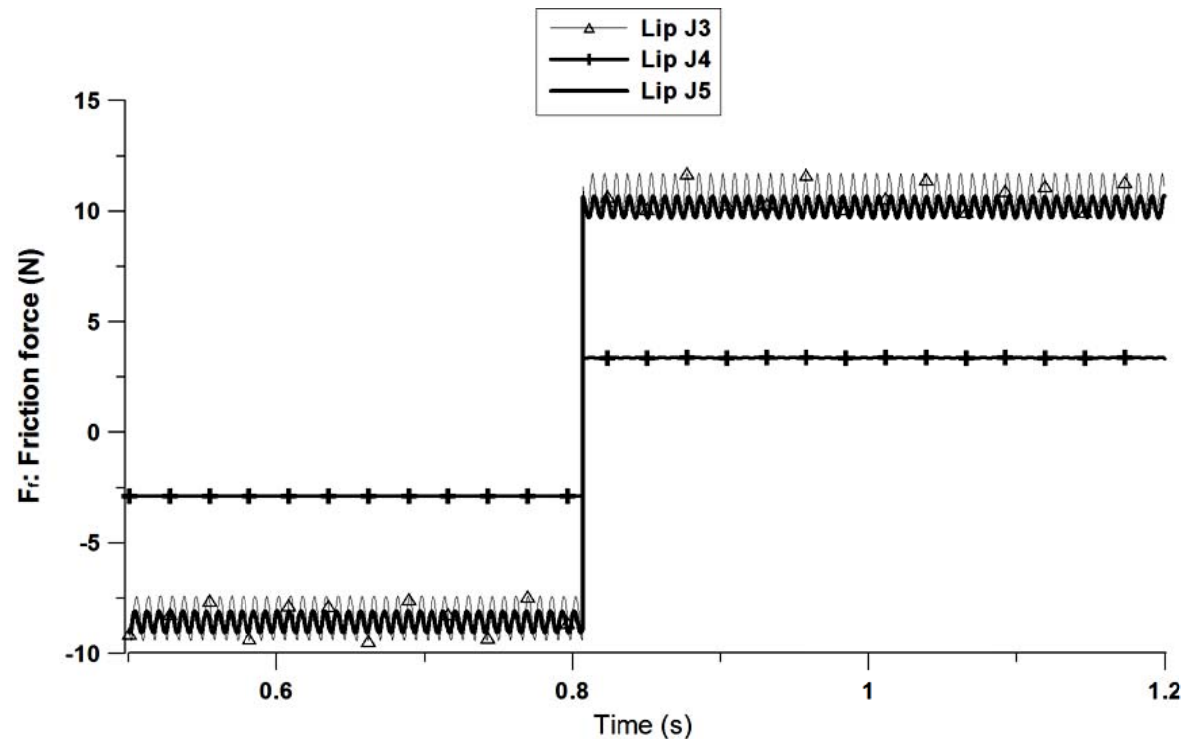

Fig.8. Friction forces in instroke and outstroke cases by varying groove shape.

It is important to note that the flow rate is given by

$$
Q=2 \pi R \rho\left[\frac{-h^{3}}{12 \mu} \frac{\partial p}{\partial x}+U \frac{h}{2}\right]_{x=0},
$$

and the fluid transport, defined as the fluid mass by integrating the flow rate $Q$ over the instroke and outstroke time.

It is demonstrated in Fig.9 that a judicious lip groove shape the seal could prevent leakage well comparing to the other forms. Therefore, with lip shape \# J4 provides a minimal friction force with weak fluid transport.

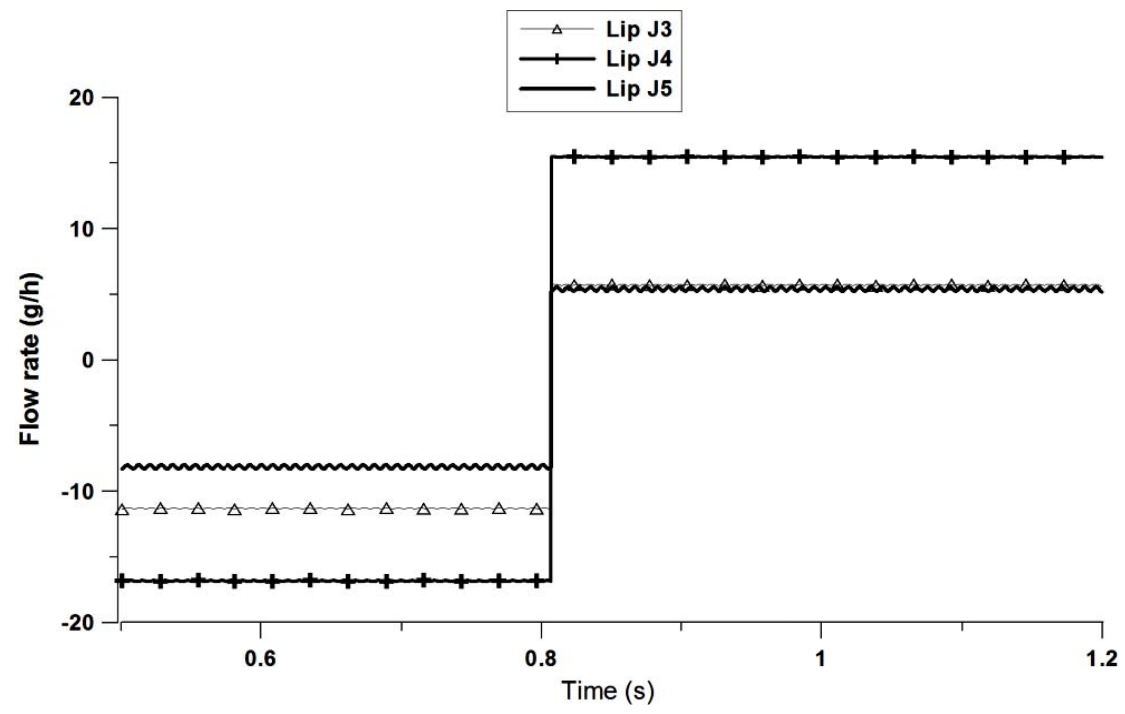

Fig.9. The flow rate in instroke and outstroke cases by varying groove shape. 


\section{Conclusion}

In this article, simulations of U-cup hydraulic rod seal were performed by using a transient EHL analysis. Throughout a one-dimensional numerical model, fluid mechanics and the elastomeric behavior of the lip were considered. The lip was assumed grooved and the rod as a rough surface.

Based on the comparisons between simulations and experiment results previously published, good agreement was underlined and showed remarkable accuracy comparing to the reverse theory.

According to the currentmodel, textured lip improves significantly the U-cup hydraulic rod seal performances. This result confirms that the technique of grooving lip is also very promising and could compete with the textured rod which is commonly known as an emerged new solution to reduce the friction effect in the sealing mechanism.

Indeed, the numerical results indicate that grooved lip effects widely sealing performances of $U$ cup reciprocating rod seal. It was proved by using a different kind of lip pattern, that the depth influences substantially the friction force comparing to the groove density effect.

However, it's necessary to note that an inappropriate groove shape could provide a high friction effect and an important flow rate.

This work opened new tracks to be investigated, namely the consideration of two grooved surfaces: lip and rod, and also the asymmetric $2 \mathrm{D}$ textured lip by including the mixed lubrication theory.

\section{Nomenclature}

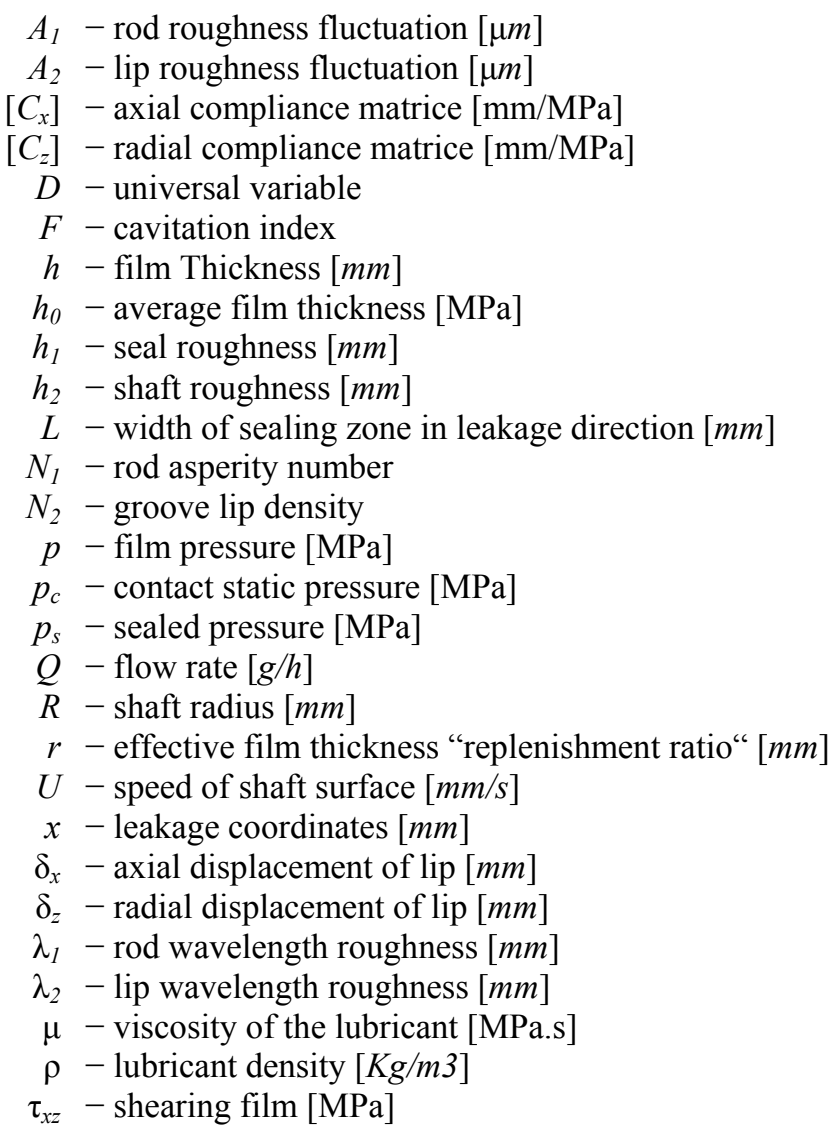




\section{References}

[1] Lawrie J.M. and O'Donoghue J.P. (1964): The mechanism of lubrication in a reciprocating seal. - Proc.2nd, The British Hydromechanics Research Association (BHRA). International Conference on Fluid Sealing, B6.

[2] Kanters A.C and Visscher M. (1989): Lubrication of reciprocating seals: experiments on the influence of surface roughness on friction and leakage. - 31th Leads-Lyon Symposium on Tribology.

[3] Crudu M. (2012): Experimental and numerical study of the Hydraulic seals. - Doctoral Thesis 2012, University of Poitiers and University of Bucarest.

[4] El Gadari M. and Hajjam M. (2017): Effect of the Grooved Rod on the Friction Force of U-Cup Hydraulic Rod Seal with Rough Lip. - Tribology Transactions, DOI:10.1080/10402004.2017.1388457

[5] Kanters A.F.C. (1990): On the calculation of leakage and friction of reciprocating elastomeric seals. - Doctoral Thesis: Technische Universiteit Eindhoven DOI: 10.6100/IR326313.

[6] Bisztray-Balku S. (1999): Tribology of elastomeric and composite reciprocating hydraulic seals. - Institute for Machine Design Technical University of Budapest H-1521 Budapest, Hungary, Periodica Polytechnica Ser. Mech. Eng., vol.43, No.1, pp.63-80.

[7] Salant R.F. and Yang B. (2008): Numerical modeling of reciprocating fluid power seals. - Proceedings of the 7th JFPS International Symposium on Fluid Power, TOYAMA 2008.

[8] Thatte A. and Salant R.F. (2009): Transient EHL analysis of an elastomeric hydraulic seal. - Tribology International, vol.42, pp.1424-1432.

[9] Elgadari M., Fatu A. and Hajjam M. (2016): Effect of grooved shaft on the rotary lip seal performance in transient condition: elasto-hydrodynamic simulations. - Tribology International, vol.93, pp.411-418.

[10] Fei Guo, Xiao Hong Jia, Longke Wang and Yuming Wang (2016): The effect of axial position of contact zone on the performance of radial lip seals with a texturing shaft surface. - Tribology International, vol.97, pp.499-508.

[11] Di Liu, Shaoping Wang, Chao Zhang and Mileta M. Tomovic (2019): Numerical study of the effects of textured shaft on the wear of rotary lip seals. - Tribology International, vol.138, pp.215-238.

[12] Kligerman Y. and Shinkarenko A. (2011): The effect of tapered edges on lubrication regimes in surface-textured elastomer seals. - Tribology International, vol.44, pp.205-2066.

[13] Yuli Huang and Salant R.F. (2016): Simulation of a hydraulic rod seal with a textured rod and starvation. Tribology International, vol.95, pp.306-315.

Received: July 25, 2019

Revised: October 1, 2019 